\title{
Unproukced Saddle Pulmonary Embolism in a Patient with Shy-Drager Syndrome: Are They Related?
}

\section{JUR}

Shahzaib Nabi' , Pushpinderdeep Kahlon' ${ }^{1}$ Tarun Jain' , Sagger Mawri' , Adeel Arshad ${ }^{2}$, Philip Kuriakose ${ }^{3}$

Department of Internal Medicine ${ }^{1}$ and Hematology-Oncology ${ }^{3}$, Henry Ford Health System, Detroit, MI 48202; ${ }^{2}$ Department of Internal Medicine, Hamad Medical Corporation, Weill Cornell University, Doha, Qatar.

Abstract:

There have been previous studies that suggested that patients with Parkinson's disease are at an increased risk of thrombosis, but could Shy-Drager syndrome, which entails Parkinsonian features, be a risk factor for thrombosis also? Our patient is a 54-year-old male with Shy-Drager syndrome who presented to the emergency department with acute onset of pleuritic chest pain and shortness of breath. Initial work-up showed normal electrocardiogram, negative troponins, normal complete blood count, and normal chemistry panel, but an elevated D-dimer. Computed tomography of the chest with intravenous contrast was performed which revealed a large saddle pulmonary embolus extending into the right and left pulmonary arteries. Interestingly, the patient remained hemodynamically stable throughout his stay in the hospital.

Key words: Shy-Drager Syndrome, Parkinson Disease, Thrombosis, Embolism, Dyspnea, Chest Pain.

\section{Introduction}

Often, when a syndrome or disease is rare, many practicing physicians do not have the in-depth information on possible implications for a patient's overall well-being. Shy-Drager syndrome is a rare entity in which a combination of Parkinson's features and autonomic impairment is clinically manifested. Specifically, Parkinson's features include tremor, bradykinesia and rigid muscles, which lead to impaired posture and balance, as well as speech changes. The additional autonomic features include orthostatic hypotension, urinary retention, incontinence or erectile dysfunction. As some studies suggest that Parkinson's, a more prevalent disease and a component of Shy-Drager syndrome, could possibly be a risk factor for developing deep vein thrombosis (DVT) and pulmonary embolism (PE), this case report attempts to provide a deeper insight into Parkinson's disease while trying to explain why this patient with Shy-Dragger syndrome might have developed an unprovoked saddle PE.

\section{Case Report}

The patient is a 54-year-old male with a past medical

\section{Corresponding Author: Dr. Shahzaib Nabi}

Email: snabi1@hfhs.org

Received: March 13,2015 | Accepted: April 17, 2015 | Published Online: April 20, 2015

This is an Open Access article distributed under the terms of the Creative Commons Attribution License (creativecommons.org/licenses/by/3.0)

Conflict of interest: None declared | Source of funding: Nil | DOl: http://dx.doi.org/10.17659/01.2015.0042 
history of Shy-Drager syndrome. He was initially diagnosed with Parkinson's disease at the age of 52. Two years later he was diagnosed with Shy-Drager syndrome by his neurologist due to a poor response to levodopa/carbidopa and episodes of severe unprovoked orthostatic hypotension, constipation, urinary retention and symptomatic bradycardia. He presented to the emergency department with right lower extremity swelling, erythema and pain. Additionally, he reported left sided pleuritic chest pain, which was sudden in onset, sharp, moderate in severity, intermittent, non-radiating, worsened with deep breathing and minimally relieved by pain medication. There was no history of travel, fever, chills, nausea, vomiting, orthopnea, paroxysmal nocturnal dyspnea, or other symptoms of heart failure or a hypercoagulable state.

Vitals showed a blood pressure of $148 / 87$ $\mathrm{mm} \mathrm{Hg}$, heart rate of 105 beats/minute, temperature of $36.7^{\circ} \mathrm{C}\left(98.1^{\circ} \mathrm{F}\right)$, respiratory rate of 20 breaths/ minute, and room air oxygen saturation of $96 \%$ on admission. Physical examination revealed a well-developed male with a mask-like face. Musculoskeletal examination was positive for right leg erythema, warmth and tenderness. Neurological examination was positive for lead-pipe type of muscle rigidity. No cardiovascular, pulmonary, abdominal, or psychiatric abnormalities were noted at the time of the examination. Social history was negative for smoking and alcohol use. There was no reported history of any movement disorders in the family. The patient's allergies included pramipexole and rotigotine. He was not taking any medications at the time of presentation.

Electrocardiogram showed sinus tachycardia. Patient's D-dimer was found to be $1540 \mathrm{ng} / \mathrm{mL}$. Computed tomography of the chest with intravenous contrast showed a large saddle PE extending into the right and left pulmonary arteries [Fig. 1-3]. Doppler ultrasound of lower extremities showed DVT of the right popliteal vein. Troponin I level was less than $0.04 \mathrm{ng} / \mathrm{mL}$ in 3 different blood draws. Complete blood count and basic metabolic panel did not show any abnormalities.

The patient was started on a weight based dose of subcutaneous enoxaparin for bridging over to warfarin starting at a $5 \mathrm{mg}$ nightly dose for at least 6 months. Reasons for longer than usual anticoagulation were the absence of any definitive provoking factors for thromboembolism (other than the patient's relative immobility from Parkinsonian features) as well as the clot burden i.e. saddle PE. The target international normalized ratio (INR) for the patient was between 2 to 3 . After the patient's INR became therapeutic, he was discharged on warfarin to complete 6 months of anticoagulation. Since discharge, the patient has been on followup with the warfarin clinic for INR management. He has also been on follow-up with the neurology and internal medicine clinics for management of Shy-Drager syndrome, but with only minimal improvement in symptoms. He is also on home physical and occupational therapy, which has been only partially helpful in controlling his progressive symptoms.

\section{Discussion}

Shy-Drager syndrome is a combination of Parkinsonism features (tremor, bradykinesia and rigid muscles) and autonomic dysfunction (orthostatic hypotension, urinary retention, incontinence or erectile dysfunction) due to central nervous system pathology making it a neurodegenerative syndrome. It is now called multiple system atrophy (MSA), with 3 different variations. MSA can be Parkinsonian feature predominant (MSA-P) due to nigrostriatal degeneration, cerebellar ataxia predominant (MSA-C) due to olivo-pontocerebellar atrophy and finally with mixed features of MSA-P as well MSA-C (MSA-M). Two-thirds of the cases of MSA are MSA-P while nearly one-third are MSA-C. A patient with MSA is more likely to present with 


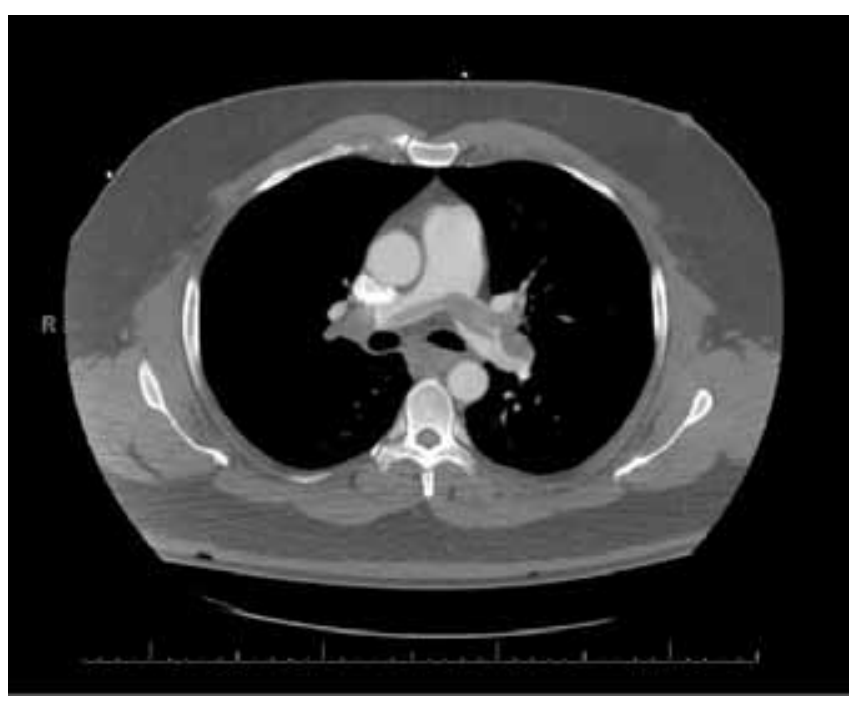

Fig.1: Large saddle pulmonary embolus at the bifurcation of main pulmonary artery.

autonomic dysfunction with symptoms of urinary retention, incontinence or erectile dysfunction, with motor dysfunction noted over the next few years. MSA has a prevalence of 2-5 cases per 1,00,000 [1]. In comparison, the prevalence of Parkinson's disease is 40 per $1,00,000$ [2]. The average age at diagnosis for MSA is 54 years [3].

According to the Second Consensus Statement on the Diagnosis of MSA [4], MSA entails orthostatic hypotension with a drop in systolic blood pressure of at least $30 \mathrm{~mm} \mathrm{Hg}$ and in diastolic blood pressure of at least $15 \mathrm{~mm} \mathrm{Hg}$ within 3 minutes of standing. Resistance to levodopa therapy is a differentiating feature of MSA diagnosis from Parkinson's disease [5]. The Unified MSA Rating Scale (UMSARS) clinical scoring system can be used to asses MSA patients and consists of 4 parts:part I consists of 12 historical items, part II consists of 14 motor examination items, part III consists of an autonomic examination and part IV consists of a global disability scale [6].

On pathological examination, there is found to be a presence of alpha-synuclien inclusions in the cytoplasm of glial cells of MSA patients [7]. There

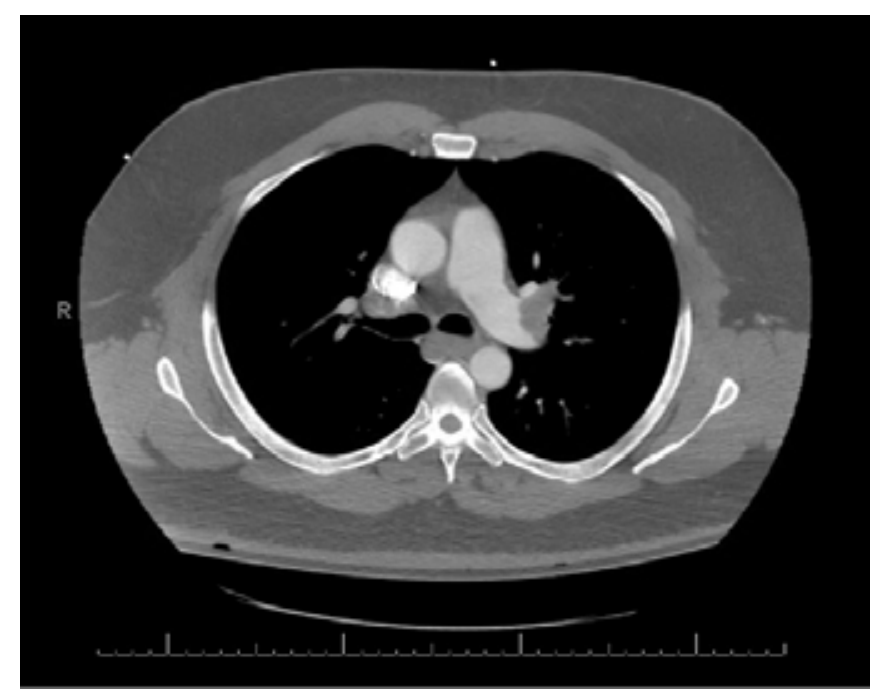

Fig.2: Large pulmonary embolus extending into the left pulmonary artery and its branches.

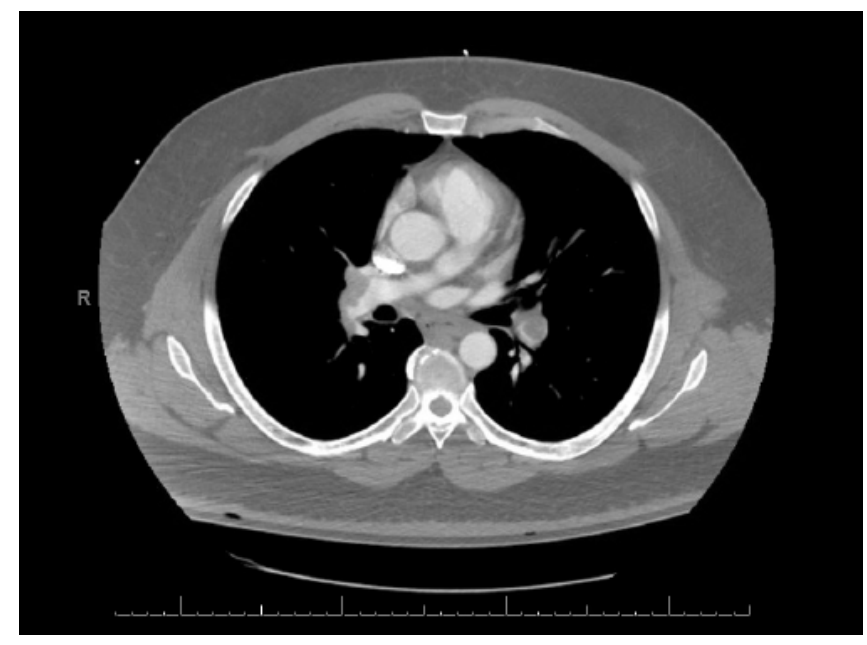

Fig.3: Massive clot burden seen in pulmonary vasculature bilaterally.

is no known blood test for the diagnosis of MSA nor is there a neuro-protective medication for its treatment. Management of the MSA patient is mostly symptomatic, including physical and occupational therapy for maintenance of mobility.

An article published in 1999 used Doppler ultrasound to examine lower extremities for 
DVTs. It suggested an increased incidence of asymptomatic DVT in nearly $5 \%$ of patients with Parkinson's disease [8]. More recently, Yamane et al. in 2013 suggested that postural abnormalities in individuals with Parkinson's disease are a risk factor for DVT. This cross-sectional study was performed using Doppler ultrasonography of the deep veins of the lower extremities in Parkinson's patients along with photographic measurement of postural abnormalities. DVTs were noted in $20 \%$ of the patients with significantly increased knee and spine bending angles. In conclusion the study indicated that Parkinson's patients have decreased mobility due to motor abnormalities, which leads to an increased risk of thrombosis [9]. In patients with Shy-Drager syndrome, in addition to decreased mobility from Parkinsonism features, another feature which might contribute to venous thromboembolism is the presence of orthostatic hypotension, which can lead to pooling and stasis of the blood in the lower extremities, thereby increasing the risk of thrombosis. Additionally, as mentioned above, patients are less responsive to levodopa treatment, which in turn can lead to more severe bradykinesia and lack of adequate mobility. This factor can also contribute to venous thromboembolism.

Even though Shy-Drager syndrome/MSA is not a common entity, one should be more vigilant of thrombosis development in such patients and be more proactive in sustaining mobility. Theoretically, such actions can lead to clinically better patient outcomes.

\section{Conclusion}

Levodopa/carbidopa treatment failure for Parkinson's disease in light of autonomic components should prompt a clinician to consider diagnosis of MSA. Shy-Drager syndrome is possibly associated with increased risk of thrombosis due to decreased mobility secondary to Parkinsonian features, as well venous stasis due to orthostasis.

\section{References}

1. Tison F, Yekhlef F, Chrysostome V, Sourgen C. Prevalence of multiple system atrophy,. Lancet. 2000;355(9202):495-496.

2. Parikh SM, Diedrich A, Biaggioni I, Robertson $D$. The nature of the autonomic dysfunction in multiple system atrophy. Journal of the Neurological Sciences. 2002;200(1-2):1-10.

3. Ben-Shlomo Y, Wenning GK, Tison F, Quinn NP. Survival of patients with pathologically proven multiple system atrophy: a meta-analysis. Neurology. 1997;48:384-393.

4. Gilman S, Wenning GK, Low PA, Brooks DJ, Mathias CJ, Trojanowski JQ, et al. Second consensus statement on the diagnosis of multiple system atrophy. Neurology. 2008;71(9):670676.

5. Kollensperger M, Geser F, Ndayisaba, JP, Boesch S, Seppi K, Ostergaard K, et al. Presentation, diagnosis, and management of multiple system atrophy in Europe: final analysis of the European multiple system atrophy registry. Movement Disorders. 2010;25(15):2604-2612.

6. Wenning GK, Tison F, Seppi K, Sampiao C, Diem A, Yekhlef F. Development and validation of the Unified Multiple System Atrophy Rating Scale (UMSARS). Movement Disorders, .2004;19(12): 1391-1402.

7. Wenning GK, Stefanova N, Jellinger KA, Poewe W, . Schlossmacher MG. Multiple system atrophy: a primary oligodendrogliopathy. Annals of Neurology.2008;64(3):239-246.

8. Burbridge BE, Wallace JK,Rajput A, McCulloch $L$, Doppler ultrasonographic examination of the leg veins of patients with Parkinson disease. Journal of Psychiatry and Neuroscience. 1999;24(4):338-340.

9. Yamane K, Kimura F, Unoda K, et al. Postural abnormality as a risk marker for leg deep venous thrombosis in Parkinson's disease. PloS One. $2013.2013 ; 8(7)$ :e66984. 